A. Barchielli and A. M. Paganoni

Nagoya Math. J.

Vol. 141 (1996), 29-43

\title{
A NOTE ON A FORMULA OF THE LÉVY-KHINCHIN TYPE IN QUANTUM PROBABILITY
}

\author{
A. BARCHIELLI AND A. M. PAGANONI
}

\section{Introduction}

In the past few years there has been an increasing interest in a certain class of stochastic differential equations (SDE's) in Hilbert spaces for applications in quantum mechanics (measurements continuous in time [1-5]) and in quantum optics (photon-detection theory and numerical simulations of master equations [6-10]). Part of the mathematical theory of these equations has been developed in [11], where also "structural properties" of this class of equations have been studied. In the paper [11] it has been shown that such equations are connected with certain semigroups of linear operators and the form of the generator of semigroups related to such SDE's has been established.

Independently of SDE's, semigroups of the same type have been studied in [12-14] and, under some mathematical restrictions, their generators have been completely classified through some quantum analogue of the Lévy-Khinchin formula. The problem is that the two forms of the generators obtained in the two papers $[11,14]$ are not directly comparable. The aim of the present note is to rewrite the generator obtained in [14] in an equivalent, more explicit form. At that point the expression for the generator of the semigroups considered in [11] will be comparable with the new form of the generator obtained in [14] and it will be evident that the semigroups studied in [11] are strictly a subclass of the ones of the article [14]. This will open the problem, at present under study, of constructing SDE's connected to semigroups with the same generality as those studied in the article [14]. This will give rise to new SDE's that haven't been taken into account in the physical literature up to now and that may have new physical applications.

Section two is dedicated to the statement of the problem and to the presentation of the results of the article [14]: semi-uniformly continuous semigroups of probability operator on von Neumann algebras and a quantum analogue of the

Received August 1, 1994. 
Lévy-Khinchin formula, giving their generator. Section three contains a technical theorem on a suitable representation of certain operator-valued measures and a second form of the quantum Lévy-Khinchin formula, which is the main result of the paper. The comparison with the generators of the semigroups obtained in [11] is also given in section three.

\section{Semigroups of probability operators}

This section deals with the introduction of the class of semigroups which we are interested in and to the presentation of the results of the article [14] on their generators. We need some preliminary notations and definitions $[15,16]$.

If $\mathcal{M}_{1}$ is a von Neumann algebra of operators on some Hilbert space $\mathscr{H}_{1}\left(\mathcal{M}_{1} \subset\right.$ $\left.\mathscr{L}\left(\mathscr{H}_{1}\right)\right)$ and $\mathscr{A} \in \mathscr{L}\left(\mathcal{M}_{1} ; \mathscr{L}\left(\mathscr{H}_{2}\right)\right)$, where $\mathscr{H}_{2}$ is another Hilbert space, the map $\mathscr{A}$ is said to be completely positive (CP) if

$$
\begin{gathered}
\sum_{i, j=1}^{n}\left\langle\phi_{i} \mid \mathscr{A}\left[a_{i}^{*} a_{j}\right] \phi_{j}\right\rangle \geq 0, \\
\forall n \in \mathbf{N}, \forall\left\{\phi_{j}, j=1, \ldots, n\right\} \subset \mathscr{H}_{2}, \quad \forall\left\{a_{j}, j=1, \ldots, n\right\} \subset \mathscr{M}_{1} .
\end{gathered}
$$

If condition (2.1) is required only for $n=1$, the map $\mathscr{A}$ is said positive. By $a^{*}$ we denote the adjoint operator of $a$. If $\mathscr{A}$ is a positive map, then $\mathscr{A}$ is said to be normal if l.u.b. $\mathscr{A}\left[a_{n}\right]=\mathscr{A}\left[\right.$ l.u.b. $\left.a_{n}\right]$ for any bounded increasing sequence $\left\{a_{n}\right\}$ of elements of $\mathcal{M}_{1} ;$ l.u.b. means least upper bound, which always exists for bounded increasing sequences in von Neumann algebras.

From now on, let $\mathscr{H}$ be a separable complex Hilbert space and denote by $\mathcal{M}:=\mathscr{L}(\mathscr{H})$ the von Neumann algebra of all bounded linear operators on $\mathscr{H}$. We need also the $W^{*}$-algebra $\mathcal{N}:=L^{\infty}\left(\mathbf{R}^{d} ; \mathcal{M}\right)$ of all essentially bounded weakly* Lebesgue-measurable functions from $\mathbf{R}^{d}$ into $\mathcal{M} ; \mathcal{N}$ is naturally isomorphic to $\mathcal{M} \otimes L^{\infty}\left(\mathbf{R}^{d}\right)$ ( $W^{*}$-tensor product) and can be seen as a von Neumann algebra of operators on the Hilbert space $\mathscr{H} \otimes L^{2}\left(\mathbf{R}^{d}\right)$. Finally, let us denote by $C_{0}\left(\mathbf{R}_{\infty}^{d}\right.$; $\mathcal{M})$ the Banach space of all norm-continuous functions from the one point compactification of $\mathbf{R}^{d}$ into $\mathcal{M}$. We use the notation $\mathbf{1}$ for the identity operator, when it is considered as an element of $\mathcal{M}, L^{\infty}\left(\mathbf{R}^{d}\right)$ or $\mathcal{N}$, and the notation Id for the identity map on $\mathcal{M}$ or $\mathcal{N}$. By $R_{z}, z \in \mathbf{R}^{d}$, we denote translations in $L^{\infty}\left(\mathbf{R}^{d}\right)$, i.e.

$$
R_{z}[f](x):=f(x+z) \text { a.e., } \forall f \in L^{\infty}\left(\mathbf{R}^{d}\right) .
$$

We are now able to introduce the semigroups we want to study. 
DEFINITION 1. A semi-uniformly continuons semigroup of probability operators (SCSPO) is a family $\left\{T_{t}, t \geq 0\right\}$ of operators $T_{t} \in \mathscr{L}(\mathcal{N})$ such that, $\forall t, s \geq 0$,

a. $T_{t}$ is $\mathrm{CP}$ and normal;

b. $T_{t} \circ\left(\operatorname{Id} \otimes R_{z}\right)=\left(\operatorname{Id} \otimes R_{z}\right) \circ T_{t}, \forall z \in \mathbf{R}^{d}$;

c. $T_{t}[1]=1$;

d. $T_{t} \circ T_{s}=T_{t+s}$;

e. $T_{0}=\mathrm{Id}$;

f. $\lim _{t \downarrow 0}\left\|T_{t}[\cdot \otimes 1]-\operatorname{Id}[\cdot] \otimes 1\right\|=0$;

g. $T_{t}[A]$ is a continuous function of $t, \forall A \in C_{0}\left(\mathbf{R}_{\infty}^{d} ; \mathcal{M}\right)$.

It has been proved [13] that properties a and $\mathrm{b}$ imply that $T_{t}$ leaves the space $C_{0}\left(\mathbf{R}_{\infty}^{d} ; \mathcal{M}\right)$ invariant, while properties $\mathrm{f}$ and $\mathrm{g}$ characterize the semi-uniform continuity [14].

The infinitesimal generator of SCSPO's involves some operator-valued measures called quasi-instruments. Let $\mathcal{M}_{*}$ be the trace-class on $\mathscr{H}$, i.e.

$$
\mathcal{M}_{*}=\left\{\rho \in \mathscr{L}(\mathscr{H}):\|\rho\|_{1} \equiv \operatorname{Tr} \sqrt{\rho^{*} \rho}<+\infty\right\} ;
$$

$\mathcal{M}_{*}$ can be identified with the predual of $\mathcal{M}$ by introducing the bilinear form

$$
\langle\rho, a\rangle:=\operatorname{Tr}\{\rho a\}, \quad \rho \in M_{*}, a \in \mathcal{M} .
$$

Let us recall that for any positive and normal map $\mathscr{A} \in \mathscr{L}(\mathcal{M})$ there exists a unique positive map $\mathscr{A}_{*} \in \mathscr{L}\left(\mathcal{M}_{*}\right)$ (the preadjoint map) such that $\langle\rho, \mathscr{A}[a]\rangle=$ $\left\langle\mathcal{A}_{*}[\rho], a\right\rangle, \forall a \in \mathcal{M}, \forall \rho \in \mathcal{M}_{*}$. On the contrary, if $\mathscr{C} \in \mathscr{L}\left(\mathcal{M}_{*}\right)$ is a positive map, then its adjoint $\mathscr{C}^{*} \in \mathscr{L}(\mathcal{M})$ (defined by $\left\langle\rho, \mathscr{C}^{*}[a]\right\rangle=\langle\mathscr{C}[\rho], a\rangle, \forall a \in \mathcal{M}$, $\left.\forall \rho \in M_{*}\right)$ is a positive and normal map.

Definition 2. A quasi-instrument on $\mathcal{M}$ with value space $(\Omega, \Sigma$ ) (a measurable space) is a map $\Gamma$ from $\sum$ into $\mathscr{L}(\mathcal{M})$ such that

a. $\Gamma(B)$ is $\mathrm{CP}$ and normal, $\forall B \in \sum$;

b. $\langle\rho, \Gamma(\cdot)[a]\rangle$ is $\sigma$-additive, $\forall \rho \in \mathcal{M}_{*}, \forall a \in \mathcal{M}$ (weak ${ }^{*} \sigma$-additivity).

If the normalization condition $\Gamma(\Omega)[1]=\mathbf{1}$ holds, $\Gamma$ is called an instrument.

Note that, if $\rho \geq 0$ and $a \geq 0,\langle\rho, \Gamma(\cdot)[a]\rangle$ is a positive finite measure. If $\Omega$ is a locally compact Hausdorff space and $\sum$ is its Borel $\sigma$-algebra, it is useful to ask such measures $\langle\rho, \Gamma(\cdot)[a]\rangle$ to have the inner and outer regularity property (as done in References [12-14]); in the case of $\Omega=\mathbf{R}^{d}$ (which is the case of interest in Definition 3), this condition is automatically satisfied by finite measures (see Theorem 2.18 p.48 in [17]).

Let us consider now the space $C_{2}\left(\mathbf{R}_{\infty}^{d} ; \mathcal{M}\right)$ of all twice differentiable functions 
such that the functions themselves and their derivatives up to second order belong to $C_{0}\left(\mathbf{R}_{\infty}^{d} ; \mathcal{M}\right)$. With the norm

$$
\|A\|_{2}:=\|A\|+\sum_{i=1}^{d}\left\|\frac{\partial A}{\partial x_{i}}\right\|+\sum_{i, j=1}^{d}\left\|\frac{\partial^{2} A}{\partial x_{i} \partial x_{j}}\right\|,
$$

$C_{2}\left(\mathbf{R}_{\infty}^{d} ; \mathcal{M}\right)$ becomes a Banach space; in Equation $(2.5)\|\cdot\|$ is the usual supremum norm in $C_{0}\left(\mathbf{R}_{\infty}^{d} ; \mathcal{M}\right)$, inherited from $L^{\infty}\left(\mathbf{R}^{d} ; \mathcal{M}\right)$. Finally, let $C_{2}\left(\mathbf{R}_{\infty}^{d}\right)$ be the analogous space of complex functions, let us set $\mathbf{R}_{*}^{d}:=\mathbf{R}^{d} \backslash\{0\}$ and denote by $\mathscr{B}_{*}$ its $\sigma$-algebra.

Definition 3. A linear map $K$ from $C_{2}\left(\mathbf{R}_{\infty}^{d} ; \mathcal{M}\right)$ into $L^{\infty}\left(\mathbf{R}^{d} ; \mathcal{M}\right)$ is said to admit a Lévy-Khinchin (LK) representation, if $K$, restricted to product elements, can be written as $\left(\forall a \in \mathcal{M}, \forall f \in C_{2}\left(\mathbf{R}_{\infty}^{d}\right), \forall x \in \mathbf{R}^{d}, \forall \rho \in \mathcal{M}_{*}\right)$

$$
\begin{gathered}
K[a \otimes f](x)=f(x) \mathscr{L}[a]+\sum_{i=1}^{d} \tilde{b}_{i} \frac{\partial f(x)}{\partial x_{i}} a+K_{1}[a \otimes f](x)+\tilde{K}_{2}[a \otimes f](x), \\
\mathscr{L}=\tilde{\mathscr{L}}_{0}+\mathscr{L}_{1}+\tilde{\mathscr{L}}_{2}, \\
\mathscr{L}_{1}[a]:=\frac{1}{2} \sum_{i, j=1}^{d} b_{i j}\left(C_{j}^{*}\left[a, C_{i}\right]+\left[C_{j}^{*}, a\right] C_{i}\right), \\
\tilde{\mathscr{L}}_{2}[a]:=\tilde{\Gamma}\left(\mathbf{R}_{*}^{d}\right)[a]-\frac{1}{2} \tilde{\Gamma}\left(\mathbf{R}_{*}^{d}\right)[1] a-\frac{1}{2} a \tilde{\Gamma}\left(\mathbf{R}_{*}^{d}\right)[1], \\
K_{1}[a \otimes f](x):=\sum_{i, j=1}^{d} b_{i j}\left\{\frac{\partial f(x)}{\partial x,}\left(C_{i}^{*} a+a C_{i}\right)+\frac{1}{2} \frac{\partial^{2} f(x)}{\partial x_{j} \partial x_{i}} a\right\}, \\
\left\langle\rho, \tilde{K}_{2}[a \otimes f](x)\right\rangle=\int_{\mathbf{R}_{*}^{d}}[f(x+y)-f(x)]\langle\rho, \tilde{\Gamma}(\mathrm{d} y)[a]\rangle \\
+\int_{\mathbf{R}_{*}^{d}} \frac{f(x+y)-f(x)}{\sqrt{\varphi(y)}\left\langle\rho, F(\mathrm{~d} y)^{*} a+a F(\mathrm{~d} y)\right\rangle} \\
+\int_{\mathbf{R}_{*}^{d}} \frac{1}{\varphi(y)}\left[f(x+y)-f(x)-\sum_{i=1}^{d} \frac{\partial f(x)}{\partial x_{i}} \frac{y_{i}}{\left.1+|y|^{2}\right] \gamma(\mathrm{d} y)\langle\rho, a\rangle,}\right. \\
\varphi(y)=\frac{|y|^{2}}{1+|y|^{2}},
\end{gathered}
$$

where $[a, b]:=a b-b a, \tilde{\mathscr{L}}_{0} \in \mathscr{L}(\mathcal{M})$ is the generator of a norm-continuous quantum dynamical semigroup [18] on $\mathcal{M},\left\{b_{i j}\right\}$ is a positive-definite $d \times d$ real matrix, $\tilde{b}_{i} \in \mathbf{R}, C_{\imath} \in \mathcal{M}, \tilde{\Gamma}$ is a quasi-instrument on $\mathcal{M}$ with value space $\left(\mathbf{R}_{*}^{d}\right.$, 
$\mathscr{B}_{*}$ ), $F$ is an $\mathcal{M}$-valued weakly ${ }^{*} \sigma$-additive measure on $\mathbf{R}_{*}^{d}$ (i.e. $F: \mathscr{B}_{*} \rightarrow \mathcal{M}$, $\langle\rho, F(\cdot)\rangle \sigma$-additive, $\left.\forall \rho \in \mathcal{M}_{*}\right), \gamma$ is a numerical finite positive measure on $\mathbf{R}_{*}^{d}$; $\tilde{\Gamma}, F$ and $\gamma$ are such that the position

$$
\begin{aligned}
\langle\rho, \Gamma(B)[a]\rangle & =\int_{B} \varphi(y)\langle\rho, \tilde{\Gamma}(\mathrm{d} y)[a]\rangle+\int_{B} \sqrt{\varphi(y)}\left\langle\rho, F^{*}(\mathrm{~d} y) a+a F(\mathrm{~d} y)\right\rangle \\
& +\langle\rho, a\rangle \gamma(B), \quad \forall B \in \mathscr{B}_{*}, \quad \forall \rho \in \mathcal{M}_{*}, \quad \forall a \in \mathcal{M},
\end{aligned}
$$

defines a quasi-instrument $\Gamma$.

Let us recall that a map $\mathscr{L}$ is the generator of a norm continuous quantum dynamical semigroup if and only if [18] $\forall a \in \mathcal{M}$

$$
\mathscr{L}[a]=\mathrm{i}[H, a]+\frac{1}{2} \sum_{k=1}^{\infty}\left(L_{k}^{*}\left[a, L_{k}\right]+\left[L_{k}^{*}, a\right] L_{k}\right),
$$

where $H, L_{k} \in \mathcal{M}, H=H^{*}, \sum_{k=1}^{\infty} L_{k}^{*} L_{k}$ is strongly convergent in $\mathcal{M}$. An equivalent form of Equation (2.14) is

$$
\mathscr{L}[a]=\mathrm{i}[H, a]+L^{*}(a \otimes 1) L-\frac{1}{2} L^{*} L a-\frac{1}{2} a L^{*} L,
$$

where $H \in \mathcal{M}, H=H^{*}$ and $L$ is a bounded linear operator from $\mathscr{H}$ into $\mathscr{H} \otimes \mathscr{K}$, with $\mathscr{K}$ an auxiliary separable Hilbert space; one goes from (2.15) to (2.14) by inserting a discrete resolution of the identity in $\mathscr{K}$.

In Definition 4 of the article [14], the LK-representation is defined by giving the action of $K$ on the whole $C_{2}\left(\mathbf{R}_{\infty}^{d} ; \mathcal{M}\right)$; however, it is sufficient to use the simpler Definition 3, given here, because of the following result.

Proposition 1. A map $K$ on $M \times C_{2}\left(\mathbf{R}_{\infty}^{d}\right)$, defined by Equations (2.6)-(2.13), can be extended by linearity and continuity to a unique continuous linear operator from $C_{2}\left(\mathbf{R}_{\infty}^{d} ; \mathcal{M}\right)$ into $C_{0}\left(\mathbf{R}_{\infty}^{d} ; \mathcal{M}\right)$.

Proof. The extension of $K$ by linearity to $\mathcal{M} \odot C_{2}\left(\mathbf{R}_{\infty}^{d}\right)$ (algebraic tensor product) is trivial. Then, we rewrite $K_{2}, \forall A \in \mathcal{M} \odot C_{2}\left(\mathbf{R}_{\infty}^{d}\right)$, as

$$
K_{2}[A](x)=\oiint_{\Gamma}\left[G_{x}\right]+\sum_{i=1}^{d} \Delta_{i}\left[\frac{\partial A(x)}{\partial x_{i}}\right]
$$

where, $\forall y \in \mathbf{R}_{*}^{d}, \forall \rho \in \mathcal{M}_{*}, \forall D \in \mathcal{M} \odot C_{0}\left\{\mathbf{R}_{\infty}^{d} \backslash\{0\}\right), \forall a \in \mathcal{M}$,

$$
G_{x}(y)=\frac{1}{\varphi(y)}\left[A(x+y)-A(x)-\sum_{i=1}^{d} \frac{\partial A(x)}{\partial x_{i}} \frac{y_{i}}{1+|y|^{2}}\right],
$$




$$
\begin{gathered}
\left\langle\rho, \mathscr{S}_{\Gamma}[D]\right\rangle=\int_{\mathbf{R}_{*}^{d}}\langle\rho, \Gamma(\mathrm{d} y)[D(y)]\rangle, \\
\left\langle\rho, \Delta_{i}[a]\right\rangle=\int_{\mathbf{R}_{*}^{d}} \frac{y_{i}}{|y| \sqrt{1+|y|^{2}}}\{\sqrt{\varphi(y)}\langle\rho, \tilde{\Gamma}(\mathrm{d} y)[a]\rangle \\
\left.+\left\langle\rho, F(\mathrm{~d} y)^{*} a+a F(\mathrm{~d} y)\right\rangle\right\} .
\end{gathered}
$$

We observe that $G_{x} \in \mathcal{M} \odot C_{0}\left(\mathbf{R}_{\infty}^{d} \backslash\{0\}\right),\left\|G_{x}\right\| \leq \lambda\|A\|_{2}$ for some $\lambda>0, \mathscr{L} \in$ $\mathscr{L}(\mathcal{M}), \Delta_{i} \in \mathscr{L}(\mathcal{M})$ for $i=1, \ldots, d,\left\|\mathscr{S}_{\Gamma}[D]\right\| \leq s\|D\|$ for some $s>0$. Then, by easy estimates one obtains $\|K[A]\| \leq \kappa\|A\|_{2}$ for some $\kappa>0$ and the unique continuous extension is guaranteed.

Given a map $K$ admitting a LK-representation, such a representation is not unique; this allows to ask the measures $F$ and $\tilde{\Gamma}$ to satisfy some additional properties (see [14] p.257).

Remark 1. Without changing $K$, it is possible to choose the measures entering Definition 3 in such a way that, for a fixed normalized vector $\phi \in \mathscr{H}$, one has, $\forall B \in \mathscr{B}_{*}, \forall g, f \in \mathscr{H}$

$$
\begin{aligned}
& \langle\phi \mid F(B) \phi\rangle=0, \\
& \langle\phi \mid \tilde{\Gamma}(B)[|\phi\rangle\langle f|] g\rangle=0, \\
& \langle f \mid \tilde{\Gamma}(B)[|g\rangle\langle\psi|] \psi\rangle=0 .
\end{aligned}
$$

Finally, the fact that $K$ determines $T_{t}$ uniquely is given by Theorem 1 and Section 7 of the article [14].

THEOREM 1. If $K$ is a linear operator on $C_{2}\left(\mathbf{R}_{\infty}^{d} ; M\right)$ that admits a $L K$-representation, then there exists a unique SCSPO such that $K$ is the restriction to $C_{2}\left(\mathbf{R}_{\infty}^{d} ; M\right)$ of its infinitesimal generator. Conversely, the restriction to $C_{2}\left(\mathbf{R}_{\infty}^{d} ; M\right)$ of the infinitesimal generator of an SCSPO admits a LK-representation.

Let us stress that the paper [14] considers the case of $d$-dimensional Lie groups instead of $\mathbf{R}^{d}$. For simplicity, this note is written only in the case of $\mathbf{R}^{d}$, but the whole content could be translated into the case of finite dimensional Lie groups. 


\section{A second form of the Lévy-Khinchin representation}

In this section we give a more convenient expression for the Lévy-Khinchin representation of the generator of a SCSPO. To do this we need some notations and a preliminary theorem on the structure of quasi-instruments.

Let $\xi$ be a (finite or $\sigma$-finite) measure on some measurable space $\left(\mathscr{Y}, \sum_{\mathscr{y}}\right)$. Let us consider the Hilbert spaces $L_{\xi}^{2}:=L^{2}(\mathscr{Y}, \xi)$ and $L_{\xi}^{2}(\mathscr{H}):=L^{2}(\mathscr{Y}, \xi ; \mathscr{H})$ of the equivalence classes of the $\xi$-square-integrable functions with values in $\mathbf{C}$ or $\mathscr{H}$, respectively. The space $L_{\xi}^{2}(\mathscr{H})$ is naturally isomorphic to $\mathscr{H} \otimes L_{\xi}^{2}$ ([15] $\S I .1 .10)$.

THEOREM 2. Let $\Gamma$ be a quasi-instrument on $\mathcal{M} \equiv \mathscr{L}(\mathscr{H})$ with value space $(\Omega, \Sigma)$; let $(\Omega, \Sigma)$ be a standard Borel space. Then, there exist a finite measure $\xi$ on $\mathscr{Y} \equiv \Omega \times \mathbf{N}$ (with the natural $\sigma$-algebra) and a bounded linear operator $V: \mathscr{H} \rightarrow$ $L_{\xi}^{2}(\mathscr{H})$, such that, $\forall h, g \in \mathscr{H}, \forall a \in \mathcal{M}, \forall B \in \sum$, one has

$$
\langle h \mid \Gamma(B)[a] g\rangle=\sum_{n=1}^{\infty} \int_{B}\langle(V h)(\omega, n) \mid a(V g)(\omega, n)\rangle \xi_{n}(\mathrm{~d} \omega),
$$

or, equivalently,

$$
\Gamma(B)[a]=V^{*}(a \otimes \chi(B)) V,
$$

where $\xi_{n}(B):=\xi(B \times\{n\})$ and $(\chi(B) u)(\omega, n):=\chi_{B}(\omega) u(\omega, n), \forall u \in L_{\xi}^{2}(\mathscr{H})$; $\chi_{B}(\cdot)$ is the characteristic function of the set $B$. On the contrary, given $\xi$ and $V$ as above, Equation (3.1) (or (3.2)) defines a quasi-instrument.

Proof. Let the quasi-instrument $\Gamma$ be given. Then, there exist a separable Hilbert space $\mathscr{K}$, a projection-valued measure $E: \Sigma \rightarrow \mathscr{L}(\mathscr{K})$, a bounded linear operator $M: \mathscr{H} \rightarrow \mathscr{H} \otimes \mathscr{K}$, such that, $\forall B \in \sum, \forall a \in M$, one has

$$
\Gamma(B)[a]=M^{*}(a \otimes E(B)) M .
$$

The proof of this statement is the same as the one given by Ozawa [19] in the case of instruments. Ozawa's proof is contained in Proposition 4.2, in the first half of the proof of Theorem 5.1 and in Corollary 5.2 of the article [19]. That proof can be repeated step by step also in the case of quasi-instruments; only at the end, in the case of instruments, $M$ turns out to be an isometry. In that proof a key role is played by the hypothesis of complete positivity of $\Gamma$; moreover, the fact that $\mathscr{K}$ can be taken separable follows from the hypothesis that $(\Omega, \Sigma)$ is a standard Borel space. 
From the spectral theorem ([20] Theorems VII.3, VIII.4) there exist a finite measure $\xi$ on $\mathscr{Y} \equiv \Omega \times \mathbf{N}$ and a unitary operator $U: \mathscr{K} \rightarrow \sum_{n=1}^{\infty}{ }^{\oplus} L^{2}\left(\Omega, \xi_{n}\right) \equiv L_{\xi}^{2}$ such that

$$
\left(U E(B) U^{*} \psi\right)(\omega, n)=\chi_{B}(\omega) \phi(\omega, n), \quad \forall \phi \in L_{\xi}^{2} .
$$

From Equations (3.3) and (3.4) we have

$$
\begin{aligned}
\Gamma(B)[a] & =M^{*}(a \otimes E(B)) M=M^{*}\left(a \otimes\left(U^{*} \chi(B) U\right)\right) M \\
& =M^{*}\left(1 \otimes U^{*}\right)(a \otimes \chi(B))(1 \otimes U) M .
\end{aligned}
$$

By defining $V:=(1 \otimes U) M$, then $V$ is a bounded linear operator from $\mathscr{H}$ into $\mathscr{H} \otimes L_{\xi}^{2} \simeq L_{\xi}^{2}(\mathscr{H})$ and Equation (3.2) is obtained. Equation (3.1) is another way of writing Equation (3.2).

Let now $\xi$ and $V$ be given; we have to prove that Equation (3.2) defines a quasi-instrument.

a. $\forall B \in \sum, \Gamma(B) \in \mathscr{L}(\mathcal{M})$; indeed, $\forall a \in \mathcal{M}$,

$$
\|\Gamma(B)[a]\|=\left\|V^{*}(a \otimes \chi(B)) V\right\| \leq\|V\|^{2}\|a\| .
$$

b. $\forall B \in \sum, \Gamma(B)$ is $\mathrm{CP}$; indeed, $\forall m \in \mathbf{N}, \forall\left\{\phi_{j}, j=1, \ldots, m\right\} \subset \mathscr{H}, \forall\left\{a_{j}\right.$, $j=1, \ldots, m\} \subset \mathcal{M}$,

$$
\begin{gathered}
\sum_{i, j=1}^{m}\left\langle\phi_{i} \mid \Gamma(B)\left[a_{i}^{*} a_{j}\right] \phi_{j}\right\rangle= \\
=\sum_{n=1}^{\infty} \int_{B}\left\langle\sum_{i=1}^{m} a_{i}\left(V \phi_{i}\right)(\omega, n) \mid \sum_{j=1}^{m} a_{j}\left(V \phi_{j}\right)(\omega, n)\right\rangle \xi_{n}(\mathrm{~d} \omega) \geq 0 .
\end{gathered}
$$

c. $\forall B \in \sum, \Gamma(B)$ is normal. Indeed, let us call $G$ the positive bounded linear map from $\mathcal{M}_{*}$ into the trace-class on $\mathscr{H} \otimes L_{\xi}^{2}$ defined by

$$
G[\rho]:=(1 \otimes \chi(B)) V \rho V^{*}(1 \otimes \chi(B)) .
$$

Then, its adjoint $G^{*}$ from $\mathscr{L}\left(\mathscr{H} \otimes L_{\xi}^{2}\right)$ into $\mathcal{M}$ turns out to be normal ([15] Lemma II.2.2). Then, $\Gamma(B)$ is the restriction of $G^{*}$ to $\mathcal{M}$ and inherits the normality property.

d. $\quad \chi(\cdot)$ is a projection-valued measure, which, in particular, is $\sigma$-additive in the strong operator topology and in the weak ${ }^{*}$ topology. By this and the fact that, $\forall \rho \in M_{*}$ and $\forall a \in M$, the quantity $V \rho V^{*}(a \otimes 1)$ is a trace-class operator on $\mathscr{H} \otimes L_{\xi}^{2}$ we have that the measure $\operatorname{Tr}_{L_{\xi}^{2}(\mathscr{H})}\left\{V \rho V^{*}(a \otimes \chi(\cdot))\right\} \equiv$ $\langle\rho, \Gamma(\cdot)[a]\rangle$ is $\sigma$-additive $\forall \rho \in \mathcal{M}_{*}, \forall a \in \mathcal{M}$. 
We are now able to give the main result.

THEOREM 3. A linear map $K$ defined on $C_{2}\left(\mathbf{R}_{\infty}^{d} ; M\right)$ admits a LK-representation if and only if its restriction to product elements can be written as $(\forall a \in \mathcal{M}, \forall f \in$ $\left.C_{2}\left(\mathbf{R}_{\infty}^{d}\right), \forall x \in \mathbf{R}^{d}, \forall h, g \in \mathscr{H}\right)$

$$
\begin{gathered}
K[a \otimes f](x)=f(x) \mathscr{L}[a]+\sum_{i=1}^{d} b_{i} \frac{\partial f(x)}{\partial x_{i}} a+K_{1}[a \otimes f](x)+K_{2}[a \otimes f](x), \\
\mathscr{L}=\sum_{i=0}^{2} \mathscr{L}_{i}, \\
\mathscr{L}_{1}[a]:=\frac{1}{2} \sum_{k=1}^{r}\left(\left[L_{k}^{*}, a\right] L_{k}+L_{k}^{*}\left[a, L_{k}\right]\right), \\
\mathscr{L}_{2}[a]=L^{*}(a \otimes 1) L-\frac{1}{2} L^{*} L a-\frac{1}{2} a L^{*} L, \\
K_{1}[a \otimes f](x):=\frac{1}{2} \sum_{i, j=1}^{d} b_{i j} \frac{\partial^{2} f(x)}{\partial x_{i} \partial x_{j}} a+\sum_{j=1}^{d} \sum_{k=1}^{r} \frac{\partial f(x)}{\partial x_{j}} a_{j k}\left(L_{k}^{*} a+a L_{k}\right), \\
=\sum_{n=1}^{\infty} \int_{\mathbf{R}_{*}^{d}}\{[f(x+y)-f(x)]\langle(L h)(y, n)+h \mid a((L g)(y, n)+g)\rangle \\
\left.-\sum_{i=1}^{d} \frac{\partial f(x)}{\partial x_{i}} \frac{y_{i}}{1+|y|^{2}}\langle h \mid a g\rangle\right\} \nu_{n}(\mathrm{~d} y),
\end{gathered}
$$

where $\mathscr{L}_{0} \in \mathscr{L}(\mathcal{M})$ is the generator of a norm-continuous quantum dynamical semigroup on $\mathcal{M}, b_{i} \in \mathbf{R},\left\{b_{i j}\right\}$ is a positive-definite $d \times d$ real matrix $\left\{a_{i j}\right\}$ is a $d \times r$ real matrix such that

$$
b_{\imath \jmath}=\sum_{k=1}^{r} a_{\imath k} a_{j k}, \quad i, j=1, \ldots, d,
$$

$\varphi$ is defined by Equation (2.12), $L_{k} \in \mathcal{M}, \nu \equiv\left\{\nu_{n}(\mathrm{~d} y)\right\}$ is a $\sigma$-finite measure on $\mathbf{R}_{*}^{d} \times \mathbf{N}$ such that

$$
\sum_{n=1}^{\infty} \int_{\mathbf{R}_{*}^{d}} \varphi(y) \nu_{n}(\mathrm{~d} y)<+\infty
$$

and $L$ is a bounded linear operator from $\mathscr{H}$ into $\mathscr{H} \otimes L_{\nu}^{2} \simeq L_{\nu}^{2}(\mathscr{H})$.

Proof. Let us start by showing that the map $K$ defined by Equations 
(2.6)-(2.13) can be written in the form (3.5)-(3.10).

Let us consider the maps $\mathscr{L}_{1}$ and $K_{1}$ defined by Equations (2.8) and (2.10). Let us fix an integer $r \geq \operatorname{rank}\left\{b_{i j}\right\}$; then, there exists a real $d \times r$ matrix $\left\{a_{i k}\right\}$ such that Equation (3.11) holds. Let us define

$$
L_{k}:=\sum_{i=1}^{d} a_{i k} C_{i}, \quad k=1, \ldots, r ;
$$

then, $L_{k} \in \mathcal{M}$ and Equations (2.8) and (2.10) can be rewritten in the form (3.7) and (3.9).

Let us consider the quasi-instrument $\Gamma$ with value space $\left(\mathbf{R}_{*}^{d} ; \mathscr{B}_{*}\right)$ introduced in Equation (2.13). By Theorem 2 there exist a finite measure $\xi$ on $\mathbf{R}_{*}^{d} \times \mathbf{N}$ and a bounded linear operator $V: \mathscr{H} \rightarrow \mathscr{H} \otimes L_{\xi}^{2} \simeq L_{\xi}^{2}(\mathscr{H})$ such that Equation (3.2) holds. Let us assume that $\tilde{\Gamma}$ and $F$ are chosen in such a way that the conditions of Remark 1 hold. Recall that in Equations (2.20) $\phi$ is a fixed vector in $\mathscr{H}$ with $\|\phi\|=1$. Under these conditions Equation (2.13) gives ( $\forall h, g \in$ $\mathscr{H}, \forall a \in \mathcal{M})$

$$
\begin{gathered}
\gamma(B)=\langle\phi \mid \Gamma(B)[|\phi\rangle\langle\phi|] \psi\rangle, \\
\int_{B} \sqrt{\varphi(y)}\langle h \mid F(\mathrm{~d} y) g\rangle=\langle\phi \mid \Gamma(B)[|\psi\rangle\langle h|] g\rangle-\langle h \mid g\rangle \gamma(B), \\
\int_{B} \varphi(y)\langle h \mid \tilde{\Gamma}(B)[a] g\rangle=\langle h \mid \Gamma(B)[a] g\rangle-\langle\phi \mid \Gamma(B)[|\psi\rangle\langle h| a] g\rangle \\
-\langle h \mid \Gamma(B)[a|g\rangle\langle\phi|] \psi\rangle+\langle h \mid a g\rangle \gamma(B) .
\end{gathered}
$$

By using Equation (3.2) and setting

$$
v(y, n):=\langle\psi \mid(V \phi)(y, n)\rangle,
$$

we obtain $v \in L_{\xi}^{2}$ and

$$
\begin{gathered}
\gamma(B)=\langle v \mid \chi(B) v\rangle_{L_{\xi}^{2},} \\
\int_{B} \sqrt{\varphi(y)}\langle h \mid F(\mathrm{~d} y) g\rangle=\langle h \otimes v \mid(1 \otimes \chi(B))(V g-g \otimes v)\rangle_{L_{\xi}^{2}(\mathscr{H})}, \\
\int_{B} \varphi(y)\langle h \mid \tilde{\Gamma}(B)[a] g\rangle=\langle V h-h \otimes v \mid(a \otimes \chi(B))(V g-g \otimes v)\rangle_{L_{\xi}^{2}(\mathscr{H})} .
\end{gathered}
$$

Let us define a linear operator $S: \mathscr{H} \rightarrow L_{\xi}^{2}(\mathscr{H})$ by

$$
(S g)(y, n)=\frac{1}{\sqrt{\varphi(y)}}[(V g)(y, n)-v(y, n) g] ;
$$


$S$ is a bounded operator because, by Equation (3.18), one has

$$
\|S g\|_{L_{\xi}^{2}(\mathscr{H})}^{2}=\left\langle g \mid \tilde{\Gamma}\left(\mathbf{R}_{*}^{d}\right)[1] g\right\rangle \leq\left\|\tilde{\Gamma}\left(\mathbf{R}_{*}^{d}\right)[1]\right\|\|g\|^{2} .
$$

Then, Equations (3.17) and (3.18) give

$$
\begin{gathered}
\langle h \mid F(B) g\rangle=\langle h \otimes v \mid(\mathbf{1} \otimes \chi(B)) S g\rangle_{L_{\xi}^{2}(\mathscr{H})}, \\
\tilde{\Gamma}(B)[a]=S^{*}(a \otimes \chi(B)) S .
\end{gathered}
$$

By using $S$, Equations (2.9) and (2.11) become

$$
\left\langle h \mid \tilde{\mathscr{L}}_{2}[a] g\right\rangle=\sum_{n=1}^{\infty} \int_{\mathbf{R}_{*}^{d}}\{\langle(S h)(y, n) \mid a(S g)(y, n)\rangle
$$

$$
\left.-\frac{1}{2}\langle(S h)(y, n) \mid(S a g)(y, n)\rangle-\frac{1}{2}\left\langle\left(S a^{*} h\right)(y, n) \mid(S g)(y, n)\right\rangle\right\} \xi_{n}(\mathrm{~d} y),
$$

$$
\begin{gathered}
\left\langle h \mid \tilde{K}_{2}[a \otimes f](x) g\right\rangle=\sum_{n=1}^{\infty} \int_{\mathbf{R}_{*}^{d}}\{[f(x+y)-f(x)] \\
\times\left\langle(S h)(y, n)+\frac{v(y, n)}{\sqrt{\varphi(y)}} h\right| a\left((S g)(y, n)+\frac{v(y, n)}{\sqrt{\varphi(y)} g)\rangle}\right. \\
\left.-\sum_{i=1}^{d} \frac{\partial f(x)}{\partial x_{i}} \frac{y_{i}}{1+|y|^{2}} \frac{|v(y, n)|^{2}}{\varphi(y)}\langle h \mid a g\rangle\right\} \xi_{n}(\mathrm{~d} y) .
\end{gathered}
$$

Let us now introduce the sets

$$
A_{n}:=\left\{y \in \mathbf{R}_{*}^{d}: v(y, n)=0\right\}, \quad A_{n}^{c}:=\mathbf{R}_{*}^{d} \backslash A_{n},
$$

which are measurable sets because $v$ is a measurable function. Moreover, we define

$$
\begin{gathered}
\nu_{n}(\mathrm{~d} y):=\left[\chi_{A_{n}}(y)+\chi_{A_{n}^{c}}(y) \frac{|v(y, n)|^{2}}{\varphi(y)}\right] \xi_{n}(\mathrm{~d} y) \\
(L g)(y, n):=\chi_{A_{n}}(y)[(S g)(y, n)-g]+\chi_{A_{n}^{c}}(y) \frac{\sqrt{\varphi(y)}}{v(y, n)}(S g)(y, n) .
\end{gathered}
$$

Equation (3.23) defines a $\sigma$-finite measure on $\mathbf{R}_{*}^{d} \times \mathbf{N}$ such that

$$
\begin{aligned}
\sum_{n=1}^{\infty} \int_{\mathbf{R}_{*}^{d}} \varphi(y) \nu_{n}(\mathrm{~d} y) & =\sum_{n=1}^{\infty} \int_{A_{n}} \varphi(y) \xi_{n}(\mathrm{~d} y)+\sum_{n=1}^{\infty} \int_{A_{n}^{c}}|v(y, n)|^{2} \xi_{n}(\mathrm{~d} y) \\
& \leq \xi\left(\mathbf{R}_{*}^{d} \times \mathbf{N}\right)+\|v\|_{L_{\xi}^{2}}^{2}<+\infty
\end{aligned}
$$

while Equation (3.24) defines a bounded linear operator $L: \mathscr{H} \rightarrow L_{\nu}^{2}(\mathscr{H})$; indeed, one has 


$$
\begin{aligned}
\|L g\|_{L_{\nu}^{2}(\mathscr{H})}^{2} & =\sum_{n=1}^{\infty} \int_{A_{n}}\|(S g)(y, n)-g\|^{2} \xi_{n}(\mathrm{~d} y)+\sum_{n=1}^{\infty} \int_{A_{n}^{c}}\|(S g)(y, n)\|^{2} \xi_{n}(\mathrm{~d} y) \leq \\
\leq & \|g\|^{2} \sum_{n=1}^{\infty} \xi_{n}\left(A_{n}\right)+\|S g\|_{L_{\xi}^{2}(\mathscr{H})}^{2} \leq\left(\xi\left(\mathbf{R}_{*}^{d} \times \mathbf{N}\right)+\|S\|^{2}\right)\|g\|^{2} .
\end{aligned}
$$

Equations (3.23) and (3.24) can be inverted to give

$$
\xi_{n}(\mathrm{~d} y)=\left[\chi_{A_{n}}(y)+\chi_{A_{n}^{c}}(y) \frac{\varphi(y)}{|v(y, n)|^{2}}\right] \nu_{n}(\mathrm{~d} y)
$$

$$
(S g)(y, n)=\chi_{A_{n}}(y)[(L g)(y, n)+g]+\chi_{A_{n}^{c}}(y) \frac{v(y, n)}{\sqrt{\varphi(y)}}(L g)(y, n) .
$$

By inserting Equations (3.25) and (3.26) into (3.21) and (3.22) one obtains

$$
\begin{aligned}
& \left\langle h \mid \tilde{\mathscr{L}}_{2}[a] g\right\rangle=\sum_{n=1}^{\infty} \int_{\mathbf{R}_{*}^{d}}\{\langle(L h)(y, n) \mid a(L g)(y, n)\rangle \\
- & \left.\frac{1}{2}\langle(L h)(y, n) \mid(\operatorname{Lag})(y, n)\rangle-\frac{1}{2}\left\langle\left(L a^{*} h\right)(y, n) \mid(L g)(y, n)\right\rangle\right\} \nu_{n}(\mathrm{~d} y) \\
+ & \frac{1}{2} \sum_{n=1}^{\infty} \int_{A_{n}}\left\{\langle(L h)(y, n) \mid a g\rangle+\left\langle a^{*} h \mid(L g)(y, n)\right\rangle\right. \\
- & \left.\langle h \mid(L a g)(y, n)\rangle-\left\langle\left(L a^{*} h\right)(y, n) \mid g\right\rangle\right\} \nu_{n}(\mathrm{~d} y),
\end{aligned}
$$

$$
\left\langle h \mid \tilde{K}_{2}[a \otimes f](x) g\right\rangle=\sum_{n=1}^{\infty} \int_{\mathbf{R}_{*}^{d}}\{[f(x+y)-f(x)]
$$

$$
\begin{gathered}
\left.\times\langle(L h)(y, n)+h \mid a((L g)(y, n)+g)\rangle-\sum_{i=1}^{d} \frac{\partial f(x)}{\partial x_{i}} \frac{y_{i}}{1+|y|^{2}}\langle h \mid a g\rangle\right\} \nu_{n}(\mathrm{~d} y) \\
+\langle h \mid a g\rangle \sum_{i=1}^{d} \frac{\partial f(x)}{\partial x_{i}} \sum_{n=1}^{\infty} \int_{A_{n}} \frac{y_{i}}{1+|y|^{2}} \xi_{n}(\mathrm{~d} y) ;
\end{gathered}
$$

let us stress that the two last lines of Equation (3.27) can be rewritten as

$$
\begin{gathered}
\frac{1}{2} \sum_{n=1}^{\infty} \int_{A_{n}}\left\{\langle(S h)(y, n) \mid a g\rangle+\left\langle a^{*} h \mid(S g)(y, n)\right\rangle-\langle h \mid(S a g)(y, n)\rangle\right. \\
\left.-\left\langle\left(S a^{*} h\right)(y, n) \mid g\right\rangle\right\} \xi_{n}(\mathrm{~d} y) .
\end{gathered}
$$

Let us define an operator $R \in \mathscr{L}(\mathscr{H})$ by

$$
\langle h \mid R g\rangle:=\sum_{n=1}^{\infty} \int_{A_{n}}\langle h \mid(S g)(y, n)\rangle \xi_{n}(\mathrm{~d} y) \equiv\langle h \otimes u \mid S g\rangle_{L_{\xi}^{2}(\mathscr{H})},
$$

where $u \in L_{\xi}^{2}$ is defined by $u(y, n)=\chi_{A_{n}}(y) ; R$ is indeed bounded because 


$$
\|R g\|^{2}=\langle(R g) \otimes u \mid S g\rangle_{L_{\xi}^{2}(\mathscr{H})} \leq\|R g\|\|u\|\|S\|\|g\|,
$$

and so

$$
\|R g\| \leq\|u\|\|S\|\|g\| .
$$

Finally, we define $\mathscr{L}_{2}$ and $K_{2}$ by Equations (3.8) and (3.10) and set

$$
\begin{gathered}
\mathscr{L}_{0}[a]=\tilde{\mathscr{L}}_{0}[a]+\mathrm{i}\left[\frac{\mathrm{i}}{2}\left(R-R^{*}\right), a\right], \\
b_{i}=\tilde{b}_{i}+\sum_{n=1}^{\infty} \int_{A_{n}} \frac{y_{i}}{1+|y|^{2}} \xi_{n}(\mathrm{~d} y) ;
\end{gathered}
$$

this completes the proof of the first part.

Let us now assume that $K$ is defined by Equations (3.5)-(3.12). It is always possible to write $L_{k}=\sum_{i=1}^{d} a_{i k} C_{i}$ for some $C_{i} \in \mathcal{M}$; then Equations (3.7) and (3.9) can be immediately written in the form (2.8) and (2.10).

Equations (3.8) and (3.9) can be rewritten in the form (2.9) and (2.11) and Equation (2.13) is satisfied by setting, $\forall h, g \in \mathscr{H}, \forall a \in \mathcal{M}, \forall B \in \mathscr{B}_{*}$,

$$
\begin{gathered}
\langle h \mid \tilde{\Gamma}(B)[a] g\rangle=\sum_{n=1}^{\infty} \int_{B}\langle(L h)(y, n) \mid a(L g)(y, n)\rangle \nu_{n}(\mathrm{~d} y) \\
\langle h \mid \Gamma(B)[a] g\rangle=\sum_{n=1}^{\infty} \int_{B}\langle(L h)(y, n)+h \mid a((L g)(y, n)+g)\rangle \varphi(y) \nu_{n}(\mathrm{~d} y) \\
\langle h \mid F(B) g\rangle=\sum_{n=1}^{\infty} \int_{B}\langle h \mid(L g)(y, n)\rangle \sqrt{\varphi(y)} \nu_{n}(\mathrm{~d} y) \\
\gamma(B)=\sum_{n=1}^{\infty} \int_{B} \varphi(y) \nu_{n}(\mathrm{~d} y)
\end{gathered}
$$

Showing that all these quantities are well defined and satisfy the requirements of Definition 3 is a standard task and it is similar to the second half of the proof of Theorem 2.

The generator obtained in [11] from the SDE approach has the form described in Theorem 3 except $K_{2}$ and the related term $\mathscr{L}_{2}$ : the expression for $K_{2}$ obtained in the paper [11] was

$$
\begin{gathered}
\left\langle h \mid K_{2}[a \otimes f](x) g\right\rangle \\
=\sum_{n=1}^{\infty} \int_{\mathbf{R}_{*}^{d}}\{[f(x+y)-f(x)]\langle(L(y, n)+1) h \mid a(L(y, n)+1) g\rangle
\end{gathered}
$$




$$
\left.-\sum_{i=1}^{d} \frac{\partial f(x)}{\partial x_{i}} \frac{y_{i}}{1+|y|^{2}}\langle h \mid a g\rangle\right\} \nu_{n}(\mathrm{~d} y),
$$

where $L(y, n) \in \mathcal{M}$, it is strongly measurable, the integral

$$
\sum_{n=1}^{\infty} \int_{\mathbf{R}_{*}^{d}} L(y, n)^{*} L(y, n) \nu_{n}(\mathrm{~d} y)
$$

is weakly convergent and

$$
\sup _{y, n}\|L(y, n)\|<+\infty
$$

It is easy to see that the equation

$$
L(y, n) h=:(L h)(y, n)
$$

defines a bounded linear operator $L$ from $\mathscr{H}$ into $L_{\nu}^{2}(\mathscr{H})$ and therefore the generators obtained in the paper [11] are in the class of generators studied in this note. The viceversa is not true: not always can Equation (3.10) be written in the form (3.36), even if the technical assumption (3.37) is not taken into account. A counterexample is as follows.

Let us fix a vecter $\varphi_{0} \in \mathscr{H}$ and take $\mathscr{H}=L_{\nu}^{2}$; then, we define $L$ by

$$
L h:=\varphi_{0} \otimes h \quad \text { or } \quad(L h)(y, n)=h(y, n) \varphi_{0} .
$$

In the general case, for instance when the measures $\nu_{n}$ are absolutely continuous with respect to Lebesgue measure, no family $L(y, n)$ of bounded operators exists such that Equation (3.38) holds. Our conjecture is that just this kind of operators (3.39) could be involved in models for "direct detection" of photons when the photons are emitted in electronic transitions from a continuoum of states to a ground state.

Acknowledgements. The authors are thankful to A. S. Holevo for the example of Equation (3.39) and for discussions on the subject.

\section{REFERENCES}

[1] V. P. Belavkin, Nondemolition measurements, nonlinear filtering and dynamic programming of quantum stochastic processes, In A. Blaquiere (ed.), Modelling and Control of Systems, Lecture Notes in Control and Information Sciences, vol.121, Springer, Berlin, 1988, pp.245-265.

[2] L. Diósi, Continuous quantum measurement and Ito formalism, Phys. Lett. A129 (1988), 419-423.

[3] A. Barchielli, V. P. Belavkin, Measurements continuous in time and a posteriori 
states in quantum mechanics, J. Phys. A: Math. Gen., 24 (1991), 1495-1514.

[4] A. Barchielli, Stochastic differential equations and 'a posteriori' states in quantum mechanics, Int. J. Theor. Phys., 32 (1993), 2221-2232.

[5] A. Barchielli, On the quantum theory of measurements continuous in time, Rep. Math. Phys., 33 (1993), 21-34.

[6] V. P. Belavkin, P. Staszewski, A continuous observation of photon emission, Rep. Math. Phys., 29 (1991), 213-225.

[7] A. Barchielli, Some stochastic differential equations in quantum optics and measurement theory: the case of counting processses. In L. Diósi, B. Lukács (ed.), Stochastic Evolution of Quantum States in Open Systems and in Measurement Processes, World Scientific, Singapore, 1994, pp.1-14.

[8] H. J. Carmichael, An Open System Approach to Quantum Optics, Lect. Notes in Physics, Series m, vol. 18, Springer, Berlin, 1993.

[9] H. M. Wiseman, G. J. Milburn, Interpretation of quantum jump and diffusion processes illustrated on the Bloch sphere, Phys. Rev. A, 47 (1993), 1652-1666.

[10] N. Gisin, P. L. Knight, I. C. Percival, R. C. Thompson, D. C. Wilson, Quantum state diffusion theory and a quantum jump experiment, J. Mod. Opt., 40 (1993), $1663-1671$.

[11] A. Barchielli, A. S. Holevo, Constructing quantum measurement processes via classical stochastic calculus, Stochastic Processes and their Applications, 58 (1995) $293-317$.

[12] A. Barchielli, Probability operators and convolution semigroups of instruments in quantum probability, Probab. Theory Rel. Fields, 82 (1989), 1-8.

[13] A. Barchielli, G. Lupieri, A quantum analogue of Hunt's representation theorem for the generator of convolution semigroups on Lie groups, Probab. Theory Rel. Fields, 88 (1991), 167-194.

[14] A. Barchielli, A. S. Holevo, G. Lupieri, An analogue of Hunt's representation theorem in quantum probability, J. Theor. Probab., 6 (1993), 231-265.

[15] E. B. Davies, Quantum Theory of Open Systems, Academic Press, London, 1976.

[16] S. Sakai, $C^{*}$-algebras, Springer, Berlin, 1971.

[17] W. Rudin, Real and Complex Analysis, McGraw-Hill, New York, 1966.

[18] G. Lindblad, On the generators of quantum dynamical semigroups. Commun. Math. Phys., 48 (1976), 119-130.

[19] M. Ozawa, Quantum measuring processes of continuous observables, J. Math. Phys., 25 (1984), 79-87.

[20] M. Reed, B. Simon, Methods of Modern Mathematical Physics. I: Functional Analysis, Academic, New York, 1972.

\section{A. Barchielli \\ Università di Milano \\ Dipartimento di Matematica \\ Via Saldini 50 \\ I-20133 Milano \\ Italy \\ and}

Istituto Nationale di Fisica Nucleare

Sezione di Milano

\author{
A. M. Paganoni \\ Università di Milano \\ Departimento di Matematica \\ Via Saldini 50 \\ I-20133 Milano \\ Italy
}

